Available online at GSC Online Press Directory

GSC Biological and Pharmaceutical Sciences

e-ISSN: 2581-3250, CODEN (USA): GBPSC2

Journal homepage: https://www.gsconlinepress.com/journals/gscbps

(RESEARCH ARTICLE)

\title{
Unfavorable progression of a subchorionic hematoma: A case report and review of the literature.
}

\author{
Sofiane Kouas ${ }^{1,}{ }^{*}$, Olfa Zoukar ${ }^{2}$, Khouloud Ikridih ${ }^{1}$, Sameh Mahdhi ${ }^{1}$, Ichrak Belghaieb ${ }^{1}$ and Anis Haddad ${ }^{2}$ \\ ${ }^{1}$ Department of Gynecology and Obstetrics, Monastir Medical School, Monastir University, Gynecology-Obstetric Service \\ Mahdia-Tunisia. \\ 2 Department of Gynecology and Obstetrics, Monastir Medical School, Monastir University, El Omrane Hospital of \\ Monastir-Monastir-Tunisia.
}

Publication history: Received on 26 July 2020; revised on 06 August 2020; accepted on 09 August 2020

Article DOI: https://doi.org/10.30574/gscbps.2020.12.2.0242

\begin{abstract}
A hematoma in the uterus or intrauterine hematoma is an effusion of blood that accumulates inside the uterine cavity during gestation. Hematomas, especially subchorionic hematomas, appear most often during the first trimester of pregnancy and can occur with or without vaginal bleeding. They are always a major cause for concern for pregnant women. We will consider pregnancy as a high risk pregnancy. It will then be necessary for the woman to keep rest and benefit from more exhaustive follow-up.
\end{abstract}

This work, carried out from a case observed in our service and from a review of the literature, aims to highlight the existence of this entity and the possible unfavorable development with fetal and maternal risks.

Keywords: Subchorionic hematoma; Ultrasound; Bleeding; Possible unfavorable course

\section{Introduction}

A hematoma in the uterus or intrauterine hematoma is an effusion of blood that accumulates inside the uterine cavity during gestation. Hematomasmost often appear during the first trimester of pregnancy. Though they are the most common cause of first trimester bleeding, they can occur with or without vaginal bleeding. A distinction must be made between hematomas in early pregnancy and those occurring in the last trimester, which involves serious risks of placental abruption. In fact, intrauterine hematomas can be classified according to their location. We will then speak about: subchorionic hematoma, retro placental hematoma and later placental hematoma.

Intrauterine hematomas are still a major cause for concern for pregnant women. Pregnancy is to be considered as a high risk pregnancy. To avoid adverse pregnancy outcomes, pregnant women are advised to take enough rest and benefit from more comprehensive follow-up care.

The appearance of a hemorrhage under the placenta or at its extremity (or behind an isolated part of the fetal membranes) raises serious concerns since the management is not well codified and the progression of this entity is unpredictable.

We report in this article the case of a patient with a subchorionic hematoma diagnosed from the start of pregnancy on ultrasound and we will try through this case report and a review of the literature to emphasize the importance of this entity whose progression may be unfavorable.

\footnotetext{
${ }^{*}$ Corresponding author: Kouas Sofiane
}

Copyright (C) 2020 Author(s) retain the copyright of this article. This article is published under the terms of the Creative Commons Attribution Liscense 4.0. 


\section{Case presentation}

A 34-year-old woman with no specific medical history, Rhesus 0 positive blood group, G3P2 with two vaginal deliveries was seen in our emergency room for persistent vaginal bleeding over an eight-week period of amenorrhea. The physical examination was without particularities except for endo-uterine bleeding of moderate abundance made of bright red clots.

Obstetric ultrasound objectified the presence of a subchorionic hematoma occupying the entire posterior wall of the uterus (Figure 1) with a trophoblast of anterior insertion; pregnancy being progressive in relation to amenorrhea.

The patient was readmitted to our department at 20 weeks of amenorrhea due to a genital hemorrhage of average abundance with the presence of a retro-chorionic hematoma of $7.3 \mathrm{~cm} \mathrm{x} 7.6 \mathrm{~cm} \times 9.2 \mathrm{~cm}$ on ultrasound.

A week later, the patient reconsulted due to massive vaginal bleeding, and biology revealed a poorly tolerated hypochromic microcytic anemia due to iron deficiency requiring the transfusion of two iso-group iso- rhesus packed red blood cells.

During the ultrasound assessment, the length of the endovaginal cervix and the amount of amniotic fluid were normal while the subchoreal hematoma increased in size. The clinical course was favorable and the patient was then discharged and followed up on an outpatient basis.

At 27 weeks of gestation, the patient was seen again after another bleeding episode accompanied by uterine contractions. The fetus was in the breech position and presented an IUGR (biometry of 25 weeks) with an amniotic index of Phelan reduced to $4.3 \mathrm{~cm}$ and a subchorial hematoma of $12 \mathrm{~cm}$ of major axis on obstetric ultrasound.

Fetal pulmonary maturation was done. The patient remained stable until 32 weeks of gestation.

Given the cataclysmic recurrence of the haemorrhage and the fetal breech presentation, an extraction by the high route for maternal rescue was indicated and a newborn male weighing $1590 \mathrm{~g}$ was delivered with an Apgar at one, five and 10 minutes of 2, 4 and 5 respectively (Figure 6). The placenta presented on gross examination a large hematoma on its placental surface (Figures 2, 3) confirmed on histology (Figures 4, 5): a reduced weight placenta (213 g) with marginal insertion of the cord 2, $3 \mathrm{~cm}$ from the nearest margin and a hematoma under massive chorion.

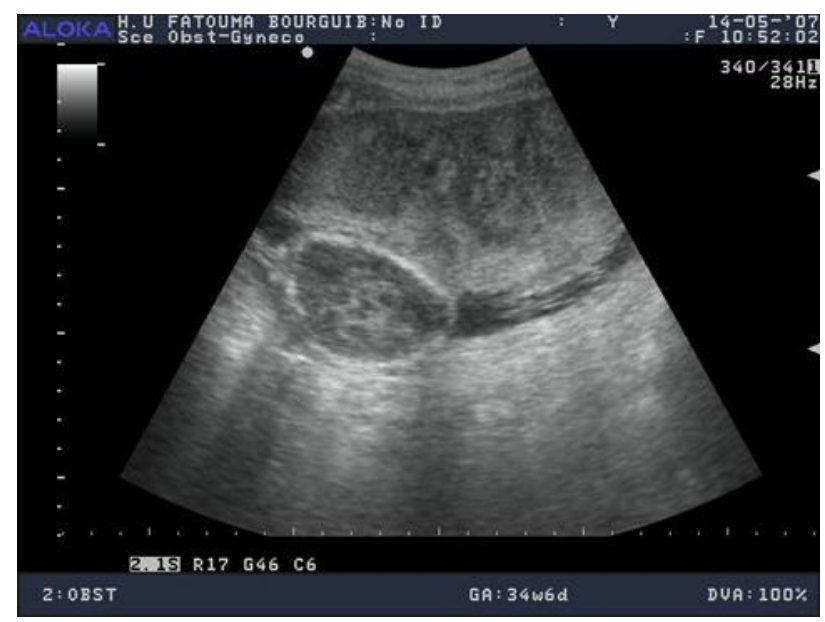

Figure 1 Ultrasound performed at 8 S. A showing the existence of a subchorionic hematoma. 


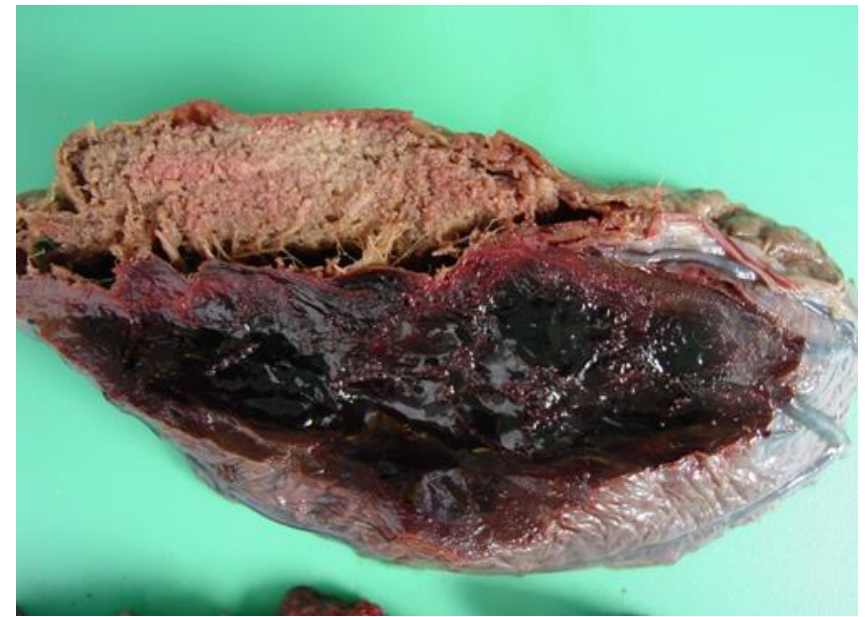

Figure 2 Macroscopic appearance of the placenta with a voluminous hematoma on the fetal side of the placenta

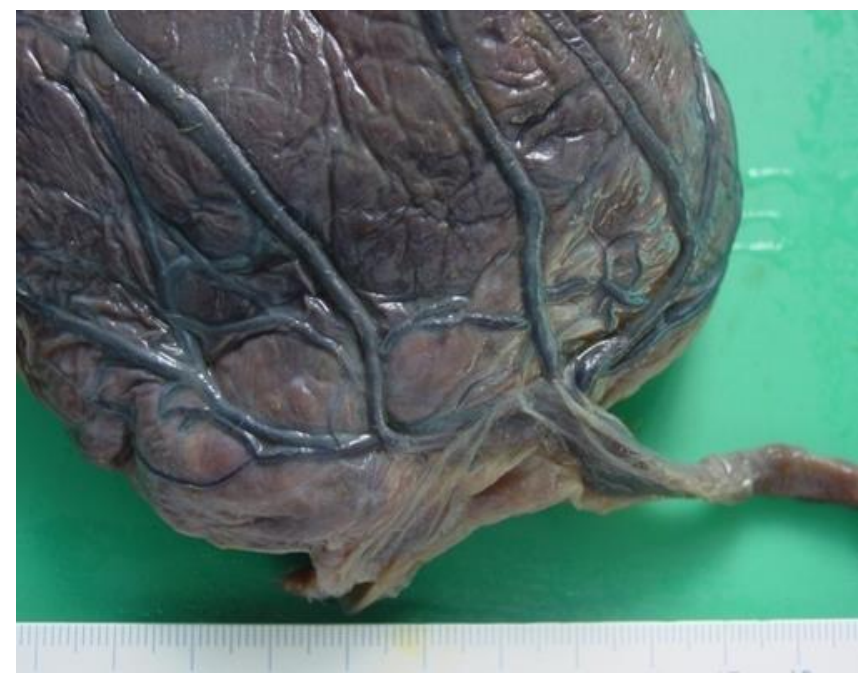

Figure 3 Macroscopic appearance of the placenta with a voluminous hematoma on the fetal side of the placenta.

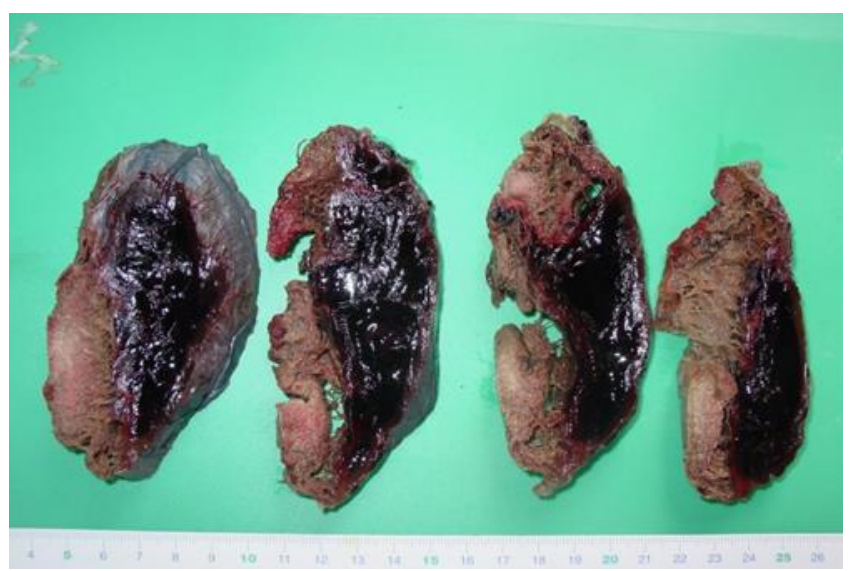

Figure 4 Appearance of a sub-chorionic hematoma at section for anatomopathological examination 


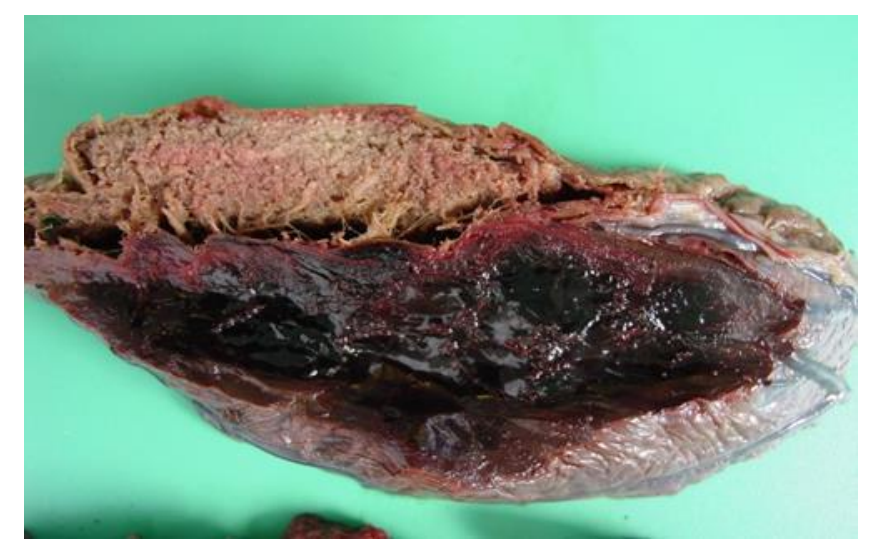

Figure 5 Appearance of a sub-chorionic hematoma at section for anatomopathological examination.

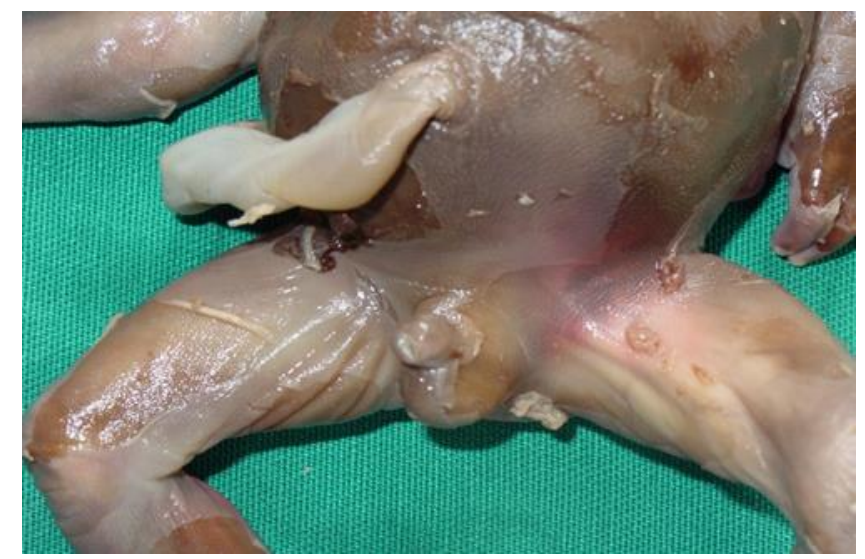

Figure 6 Male fetus from a pregnancy complicated by a massive subchorionic hematoma with the presence of multiple bubbles under the skin.

\section{Discussion}

The incidence of subchorial hematoma is difficult to assess due to the poor literature, but it is thought to vary greatly from 4 to $48 \%$ depending on the study [3].

Otherwise; the etiopathogenic mechanism behind these hematomas remains uncertain and poorly understood. The causes of the formation of intrauterine hematomas are not known. However, their appearance has been associated with the following factors: bad habits such as smoking or alcoholuse, pre-existing abdominal lesions, placenta previa, hypertension or pre-eclampsia, numerous prior pregnancies, and being aged over 38-40 years etc.

Most likely, intrauterine hematomas originate at the time of implantation or embryonic implantation, due to the damage caused in the endometrium which ultimately turns into bloody effusion. If the blood clots do not resolve, the hematomas can continue to increase and put more and more pressure on the embryo, causing spontaneous abortion in the worst case.

The clinical picture resulting from placental abruption is extremely variable and depends on several factors such as the site and size of the hematoma, gestational age, chronic bleeding and the existence or not of underlying diseases [1].

If the bleeding comes from the spiral arteries during the retro placental hematoma, it comes from the veins at the margin of the placenta in case of subchorionic hematoma. 
At gray scaleUS, it was thought that hematomas on the fetal side of the placenta could not be distinguished from chorioangiomas [2]. With the advent of color Doppler, the abundant blood flow which would be visualized in the event of chorioangioma would be absent in a subchorionic hematoma.

Due to certain diagnostic difficulties, placental MRI could be useful for the diagnosis.

Some authors have reported an association between subchorionic hematoma and the presence of autoantibodies such as anti-cardiolipin antibodies and lupus anticoagulants [4], but this association was not objectified in our case since the immunological assessment was found to be negative.

The size and location of the subchorionic hematomas are the two main prognostic factors and considerably influence the rates of fetal loss $[5,6]$.

In fact, poorly located hematomas, particularly central and large, are usually accompanied by miscarriages and abortions, while small hematomas generally develop favorably and end in spontaneous resorption. The elimination time will be more or less long depending on the size and location of the hematoma. .

Fetal loss rates can be high, as high as $25 \%$ to $50 \%$ in some studies $[2,6]$. This is in agreement with another retrospective study, which found that persistent hematoma with clinical symptoms, such as vaginal bleeding and / or uterine contractions are likely to lead to an abortion or to induce premature labor as noted in our case [7].

The importance of other factors on the risk of preterm delivery, such as gestational age at detection, maternal age and parity, remains unclear.

The optimal clinical management of these patients is not well codified. However, it is clear that when these placental hematomas are massive, careful monitoring is necessary. Preventive measures will have to be taken, for example: absolute rest, sexual abstinence, progesterone tablets in some cases, and emergency hospitalization in the most serious cases (especially hemorrhagic).

Some authors have suggested that in the event of the loss of fetal circulation detected by Doppler ultrasound, an immediate cesarean should be performed [8]. In our case, the state of the fetus remained reassuring throughout the prenatal follow-up and a premature delivery was finally necessary for maternal indications. Other factors can also contribute to a good prognosis including strict rest, administration of corticosteroids and judicious use of tocolysis.

These pregnancies are considered to be at high risk of fetal loss and premature delivery, especially in case of bleeding episodes.

\section{Conclusion}

Chorionic intrauterine hematomas can seriously harm maternal health, especially when they are massive and bulky with or without bleeding. Ultrasound evaluation of the hematoma remains useful and makes it possible to define the perinatal prognosis.

Despite a spectacular initial presentation, this entity may prove compatible with a favorable outcome, but maternal and fetal complications remain possible.

\section{Compliance with ethical standards}

\section{Acknowledgments}

We thank all the authors who participated in this work which will serve as a reference for making future appropriate decisions.

\section{Disclosure of conflict of interest}

The authors declare no conflict of interest. 


\section{Statement of informed consent}

Informed consent was obtained from all individual participants included in the study.

\section{References}

[1] Shanklin DR and Scott JS. (1975). Massive subchorialthrombohaematoma (Breus'mole).Br J ObstetGynaecol, 82, 476-87.

[2] Richards DS and Bennett BB. (1998). Prenatal ultrasound diagnosis of massive subchorionic thrombohematoma. Ultrasound ObstetGynecol, 11, 364-6.

[3] Bromley B and Benacerraf BR. (1994). Solid masses on the fetal surface of theplacenta: differential diagnosis and clinical outcome. J Ultrasound Med, 13, 883-6.

[4] Bennett GL, Bromley B, Lieberman E and Benacerraf BR. (1996). Subchorionichemorrhage in first-trimester pregnancies: prediction of pregnancyoutcome with sonography. Radiolology, 200, 803-6.

[5] Mantoni M and Pedersen JF. (1981). Intrauterine haematoma.An ultrasonic studyof threatened abortion. Br J ObstetGynecol, 88, 47-51.

[6] Glavind K, Nohr S, Nielson PH and Ipsen L. (1991). Intra-uterine hematoma inpregnancy. Eur J ObstetGynecolReprodBiol, 40, 7-10.

[7] Seki H, Kuromaki K, Takeda S and Kinoshita K. (1998). Persistent subchorionichematoma with clinical symptoms until delivery. Int J GynecolObstet, 63, 123-8.

[8] Thomas D, Makhhoul J and Muller C. (1992). Fetal growth retardation dueto massive subchorionicthrombohematoma: report of two cases. JUltrasound Med, 11, 245-7.

\section{How to cite this article}

Kouas S, Zoukar O, Ikridih K, Mahdhi S, Belghaieb I and Haddad A. (2020). Unfavorable progression of a subchorionic hematoma: a case report and review of the literature. GSC Biological and Pharmaceutical Sciences, 12(2), 74-79. 